Publ. RIMS, Kyoto Univ.

12 Suppl. (1977), 481-490.

\title{
$S$-matrix and Renormalization
}

by

\author{
O. I. ZAVIALOV*
}

When dealing with any object in quantum field theory one inevitably encounters the problem of renormalization. The aim of the present paper is to exhibit explicitly the combinatorial structure of renormalization. This will be done on the "whole" of the perturbation expansion rather than on individual graphs [7]. We'll show further how the corresponding structure formula lead directly to such relations as Wilson expansion [1], Zimmermann identities [2], renorm-group equation, Callan-Symanzik equation [3] etc.

As far as individual Feynman graph is concerned the convenient way to renormalize it is to use Speer analytic regularization [4] or dimensional regularization [5]. Being a powerful tool in calculations these procedures still permit rather little control over the subtraction point. Since the latter imposes strong restrictions on the theory, we begin with the original version of renormalization given by Bogolubov and Parasiuk [6].

\section{$\S 1$. Notations and Bogolubov-Parasiuk Renormalization}

Let $\langle V, L\rangle$ be the Feynman graph, $V$ being a set of vertices, $L$ a set of inner lines. Then the $R$-operation is defined on the graph $\langle V, L\rangle$ by

$$
R_{\langle V, L\rangle}=\sum_{V=V_{1}+\cdots+V_{n}} \Lambda\left(V_{1}\right) \cdots \Lambda\left(V_{n}\right)
$$

where the sum is over all possible decompositions of $V$ into the nonempty and nonoverlapping subsets $V_{1}, \cdots, V_{n}$. Operators $\Lambda$ are defined by recurrent relations

$$
\Lambda\left(V_{i}\right)=\hat{I} ; \quad \text { if }\left|V_{i}\right|=1
$$

Received April 22, 1976.

* Steklov Institute for Mathematics, Moscow. 


$$
\Lambda\left(V_{i}\right)=-M\left(V_{i}\right)^{\prime} R\left(V_{i}\right)+P\left(V_{i}\right), \text { if }\left|V_{i}\right|>1
$$

where

$$
' R=\sum_{\substack{V_{i}=V_{i_{1}++\ldots+V_{i n}} V_{i_{j} \neq V_{i}} \\ i_{i n}}} \Lambda\left(V_{i_{1}}\right) \cdots \Lambda\left(V_{i_{n}}\right)
$$

The sum is over all decompositions of $V_{i}$ into nonempty nonoverlapping subsets $V_{i j} \Subset V_{i}$. The operators $M\left(V_{i}\right), P\left(V_{i}\right)$ are nonzero only if $\left\langle V_{i}, L_{i}\right\rangle$ is proper and posesses a nonnegative superficial divergence $\omega_{i}$. An operator $M\left(V_{i}\right)$ changes the coefficient function (which is supposed to be regularized via some intermediate regularization) of the graph $\left\langle V_{i}, L_{i}\right\rangle$ into a polynomial of degree $\omega_{i}$ equal to the sum of the first terms of its MacLaurin series in the external momenta. $P\left(V_{i}\right)$ is an operator of finite renormalization changing the coefficient function of $\left\langle V_{i}\right.$, $L_{i}>$ into a fixed polynomial of degree $\omega_{i}$.

We define now $R,{ }^{\prime} R, \Lambda$ and $M$ operations on arbitrary $T$-ordered function $\Phi$ of local quantities assuming that $\Phi$ can be expanded into a sum of Feynman graphs and on each of these graphs $R,{ }^{\prime} R, \Lambda$ and $M$ operate according to the rules given above (e.g. renormalized $S$-matrix is $R T \exp i \int \mathcal{L}(x) d x$, with $\mathcal{L}(x)$ interaction Lagrangian). Next we remind the notion of a composite field $B_{\{\mu\}}^{a}(x)$ introduced by Zimmermann [2]. Let $\{\mu\}$ be a multi-indix

$$
\{\mu\}=\left\{\left(\mu_{1}\right) \cdots\left(\mu_{m}\right)\right\}=\left\{\left(\mu_{10} \mu_{11} \mu_{12} \mu_{13}\right) \cdots\left(\mu_{m 0} \mu_{m 1} \mu_{m 2} \mu_{m 3}\right)\right\} .
$$

Let $\left|\mu_{j}\right|=\mu_{j 0}+\mu_{j 1}+\mu_{j 2}+\mu_{j 3}$ and $\left(\mu_{j}\right) !=\mu_{j 0} ! \cdots \mu_{j 3}$ ! Denote by $b_{\{\mu\}}(x)$ the normal monomial of the free field $\varphi(x)$

$$
b_{\{\mu\}}(x) \equiv: \varphi_{\left(\mu_{1}\right)}(x) \cdots \varphi_{\left(\mu_{m}\right)}(x): \equiv: \partial_{\left(\mu_{1}\right)} \varphi(x) \cdots \partial_{\left(\mu_{m}\right)} \varphi(x): .
$$

Let $d=m+\sum\left|\mu_{i}\right|$ be a dimension of this monomial, and $a$ an integer $a \geqq d$. Define a formal $T$-product $T b_{\{\mu\}}(x) \exp i \int \mathcal{L}\left(x^{\prime}\right) d x^{\prime}$ and prescribe to any Feynman graph $\left\langle V_{i}, L_{i}\right\rangle$ in the perturbation expansion of this formal expression an index $\omega_{i}$ according to the following rules

$$
\begin{array}{ll}
\omega_{i}=a-l_{i} & \text { if } x \in V_{i} \\
\omega_{i}=4-l_{i} & \text { if } x \notin V_{i}
\end{array}
$$

where $l_{i}$ is the number of external lines of $\left\langle V_{i}, L_{i}\right\rangle$ (we have restricted ourselves to the case of renormalizable scalar theory). Let $R^{a}, \Lambda^{a}, M^{a}$, 
etc. be the operators (1) - (4) constructed according to (5) and corresponding to the zero finite renormalization $P^{a} \equiv 0$. Then up to nonessential operator factor the composite field is defined by

$$
B_{\{\mu\}}^{a}(x)=R^{a} T b_{\{\mu\}}(x) \exp i \int \mathcal{L}\left(x^{\prime}\right) d x^{\prime} .
$$

Relations between composite fields corresponding to different $a$ are given by Zimmermann identities (see sec. 3). Note that there are many other equally natural ways to define a composite field. For example, there is no reason why we should use the special case of finite renormalization $P^{a} \equiv 0 \cdot P^{a}$ may be considered as an operator which controls a subtraction point of the $R$-operation. It means that with some non-zero $P$ the $R$ operation can be regarded as having the structure given by (1)-(4) with $P=0$, but $M$ redefined in the sense that the center of expansion in the powers of invariant combinations of external momenta $\left(P_{i} P_{j}\right)$ is shifted from zero to some point $\overline{\left(P_{i} P_{j}\right)}$. Relations between composite fields corresponding to different subtraction points can be derived along the same lines as in sec. 3 .

At last we introduce the following short notations

$$
\begin{aligned}
& S \equiv i \int \mathcal{L}(x) d x ; \quad E_{0}(S) \equiv T \exp i \int \mathcal{L}(x) d x \\
& E_{1}(S) \equiv E_{0}(S)-1 ; \quad E_{2}(S) \equiv E_{0}(S)-1-S .
\end{aligned}
$$

In the following all the products of the local operators are supposed to be $T$-ordered, so we'll systematically omit the symbol " $T$ ", e.g.,

$$
\begin{aligned}
& R T \exp i \int \mathcal{L}(x) d x \equiv R E_{0}(S) \\
& B_{\{\mu\}}^{a}(x) \equiv R^{a} E_{0}(S) b_{\{\mu\}}(x), \text { etc. }
\end{aligned}
$$

\section{$\S 2$. Structure Formula}

Let a "field-like" object $\Phi(x)$ be expanded into a certain sum of Feynman diagrams

$$
\Phi(x)=\sum_{l} \frac{1}{l !} \int d x_{1} \cdots d x_{l} f_{l}\left(x \mid x_{1} \cdots x_{l}\right): \varphi\left(x_{1}\right) \cdots \varphi\left(x_{n}\right):
$$

with translation-invariant $f_{l}$. Note that $R^{a} E_{0}(S) b_{\{\mu\}}(x), E_{1}(S) b_{\{\mu\}}(x)$, 
$E_{1}(S) M^{a} E_{1}(S) b_{\{\mu\}}(x)$ etc. are the objects of the type (7). According to the definition of sec. 1 the operator $M^{a}$ transforms $\Phi(x)$ into

$$
M^{a} \widehat{\varphi}(x)=\sum_{l+\Sigma ; \lambda_{i} \leq a} K^{\{\lambda\}} b_{\{\lambda\}}(x)
$$

where in the case of zero subtraction point

$$
K^{\{\lambda\}}=\frac{(-i)^{\Sigma\left|\lambda_{k}\right|}}{l !\left(\lambda_{1}\right) ! \cdots\left(\lambda_{2}\right) !}\left\langle\Phi(0) \widetilde{\varphi}^{\left(\lambda_{1}\right)}(0) \cdots \widetilde{\varphi}^{\left(\lambda_{2}\right)}(0)\right\rangle^{\text {prop }} .
$$

In (9) we have used the notations of [2]. One can easily write the similar relations for the case of an arbitrary subtraction point and for the objects like $M E_{2}(S), M E_{2}(S) M E_{2}(S)$ etc. (see sec. 5).

Our task now is to express the renormalized $S$-matrix $R E_{0}(S)$ and the composite field $B(x)=R E_{0}(S) b(x)$ directly in terms of operation $M$ avoiding thus the complicated combinatorics of (1)-(4).

Lemma 1. In the case of zero finite renormalization $(P \equiv 0)$

$$
R E_{0}(S)=E_{0}\left(S-M E_{2}\left(S-M E_{2}(S-\cdots)\right)\right)
$$

We omit the proof of (10) which is nothing else than a simple algebraic manipulation with (1)-(4) (see [8]). What has happened can be explained by a trivial remark that (on the level of some intermediate regularization) we may describe the $R$-operation as a result of insertion of additional "counterterms" vertices into the initial Lagrangian, the "naive" Wick rules being unchanged. Then the renormalized Lagrangian $\mathcal{L}_{r}(x)$ is defined by

$$
i \int \mathcal{L}_{r}(x) d x=S-M E_{2}(S-\cdots) \equiv S_{r}
$$

where $S=i \int \mathcal{L}(x) d x$ coincides with "bare" interaction, $-M E_{2}\left(S-M E_{2}\right.$ $(S-\cdots))$ representing “counterterms". Though (10) defines $R E_{0}(S)=$ $E_{0}\left(S_{r}\right)$ through an infinite process, in any real calculation we can cut this process on some finite step. So (10) cut on the $N$-th step reproduces all the renormalized graphs up to $(N+1)$-th order. On the other hand for the calculation of the one-loop graphs (in any order) it is enough to expand $E_{0}\left(S-M E_{2}(S)\right)$, for two-loops graphs $-E_{0}\left(S-M E_{2}(S-\right.$ $\left.\left.M E_{2}(S)\right)\right)$ etc. Equation (10) provides a simple proof of causality and uni- 
tarity of the renormalized $S$-matrix. One has to demonstrate only the locality of the renormalized Lagrangian (which is trivially seen in (11)) and the anti-hermiticity of the "counterterm" part of the action $S_{r}-S$ (which is established by passing to Euclidean region of integration over internal momenta in the corresponding Feynman diagrams).

Now we deduce similar result for the composite field $B_{\{\mu\}}(x)$. We have

Lemma 2. [8]

$$
B_{\{\mu\}}(x)=E_{0}\left(S_{r}\right)^{\prime} B_{\{\mu\}}(x)
$$

where

$$
{ }^{\prime} B_{\{\mu\}}(x)=\frac{1}{1+M E_{1}\left(S_{r}\right)} b_{\{\mu\}}(x) .
$$

Equations (10), (12) and (13) leads to several useful consequences.

\section{§ 3. Zimmermann Identities}

Let $M^{\mathrm{I}}, M^{\mathrm{II}}$ be two different subtraction operators and $B_{\{\mu\}}^{\mathrm{I}}(x)$, $B_{\{\mu\}}^{\mathrm{I}}(x)$ and ' $B_{\{\mu\}}^{\mathrm{I}}(x),{ }^{\prime} B_{\{\mu\}}^{\mathrm{I}}(x)$ corresponding composite fields constructed according to (6) and (12). Suppose that $M^{\mathrm{I}}$ and $M^{\mathrm{II}}$ are different only on the subgraphs containing a vertex $x$, so that "inner" $R$-operations $R^{I}$ and $R^{I I}$ coincide, which means that renormalized Lagrangians $\mathcal{L}_{r}{ }^{\mathrm{I}}(x)$ and $\mathcal{L}_{r}{ }^{\mathbb{I}}(x)$ are equal: $S_{r}{ }^{\mathrm{I}}=S_{r}{ }^{\mathbb{I}}=S_{r}$. Suppose that ${ }_{\mathrm{b}}^{\mathrm{I}} M^{\mathrm{I}}, M^{\mathrm{I}}$ transform the coefficient function of the graph containing $x$-vertex into polynomials of degree $a^{I}-l, a^{I I}-l$ resp. ( $l$ is the number of external lines). Let the following condition take place

$$
M^{\mathrm{I}} M^{\mathrm{I}}=M^{\mathrm{I}}
$$

which holds for example if $a^{\mathrm{I}} \geqq a^{\mathbb{I}}$.

Now let us find a relation between the fields $B^{\mathrm{I}}$ and $B^{\text {II }}$. Using (13) we obtain

$$
{ }^{\prime} B_{\{\mu\}}^{\mathrm{I}}-{ }^{\prime} B_{\{\mu\}}^{\mathrm{I}}=\frac{1}{1+M^{\mathrm{I}} E_{1}\left(S_{r}\right)}\left(M^{\mathrm{I}}-M^{\mathrm{I}}\right) E_{1}\left(S_{r}\right) \frac{1}{1+M^{\mathrm{I}} E_{1}\left(S_{r}\right)} b_{\{\mu\}} .
$$

Due to (14) and to equations $M^{I} b_{\{\mu\}}=M^{I I} b_{\{\mu\}}=b_{\{\mu\}}$ the $E_{1}\left(S_{r}\right)$ in the 
numerator of (15) can be replaced by $E_{0}\left(S_{r}\right)$. Thus

$$
' B_{\{\mu\}}^{\mathrm{I}}(x)-{ }^{\prime} B_{\{\mu\}}^{\mathrm{I}}(x)=\frac{1}{1+M^{\mathrm{I}} E_{1}\left(S_{r}\right)}\left(M^{\mathrm{I}}-M^{\mathrm{I}}\right) B_{\{\mu\}}^{\mathrm{I}}(x) .
$$

If both $M^{\mathrm{I}}$ and $M^{\mathrm{II}}$ correspond to zero subtraction point in the subgraphs containing $x$-vertex we can use (8) and (9) to calculate $\left(M^{\mathrm{I}}-M^{\mathrm{II}}\right)$ $\times B_{\{\mu\}}^{\mathrm{I}}(x)$. We have

$$
{ }^{\prime} B_{\{\mu\}}^{\mathrm{I}}(x)-B_{\{\mu\}}^{\mathrm{I}}(x)=\sum_{a^{\mathrm{II}}<l+\sum\left|\lambda_{i}\right| \leqq a^{\mathrm{I}}} K_{\{\mu\}}^{\{\lambda\}} \frac{1}{1+M^{\mathrm{I}} E_{1}\left(S_{r}\right)} b_{\{\lambda\}}(x) .
$$

Multiplying this equation by $E_{0}\left(S_{r}\right)$ from the left and using (13), (12) and (9) we come to the Zimmermann identities:

$$
\begin{aligned}
B_{\{\mu\}}^{\mathrm{I}}(x)=B_{\{\mu\}}^{\mathrm{I}}(x) & +\sum_{a^{\mathrm{II}}<l+\Sigma\left|\lambda_{i}\right| \leqq a^{\mathrm{I}}} \frac{(-i)^{\Sigma\left|\lambda_{i}\right|}}{l !\left(\lambda_{1}\right) ! \cdots\left(\lambda_{l}\right) !} B_{\{\lambda\}}^{\mathrm{I}}(x) \\
& \times\left\langle B_{\{\mu\}}^{\mathrm{I}}(0) \widetilde{\varphi}^{\left(\lambda_{1}\right)}(0) \cdots \widetilde{\varphi}^{\left(\lambda_{l}\right)}(0)\right\rangle^{\text {prop }} .
\end{aligned}
$$

The similar relations can be obtained for $M^{\mathrm{I}}, M^{\text {II }}$ corresponding to any subtraction point if condition (14) holds [8].

\section{§4. Wilson Expansion [9]}

According to Wilson [1] the singularities (at $x_{1} \rightarrow x, \cdots, x_{n} \rightarrow x$ ) of the product of local Heisenberg fields $\varphi_{1}\left(x_{1}\right), \cdots, \varphi_{n}\left(x_{n}\right)$ are given by the relation

$$
\varphi_{1}\left(x_{1}\right) \cdots \varphi_{n}\left(x_{n}\right)=\sum_{l} Q_{l}\left(x_{1}, \cdots, x_{n}\right) B_{l}(x)+B\left(x_{1}, \cdots, x_{n}\right) .
$$

Here $B\left(x_{1}, \cdots, x_{n}\right)$ is a regular (oprator-valued) function which vanishes at $x_{1}=\cdots=x_{n}=x ; B_{l}(x)$ are local operators, and $Q_{l}\left(x_{1}, \cdots, x_{n}\right)$ is a $c$ number distributions, depending only on relative coordinates $x_{i}-x_{j}$ and singular at $x_{1}=\cdots=x_{n}=x$. In perturbation theory Wilson expansion has been checked by Zimmermann [1]. Here we give the proof of this expansion based on the structure formula (12) (13).

Let $\varphi_{\{\nu\}}\left(x_{1}, \cdots, x_{n}\right)$ be the truncated $T$-product of fields

$$
\varphi_{\{\nu\}}\left(x_{1}, \cdots, x_{n}\right) \equiv E_{0}\left(S_{r}\right): \varphi_{\left(\nu_{1}\right)}\left(x_{1}\right) \cdots \varphi_{\left(\nu_{n}\right)}\left(x_{n}\right): .
$$

Since up to a constant operator factor which vanishes in vacuum expectation value the product $\varphi_{1}\left(x_{1}\right) \cdots \varphi_{n}\left(x_{n}\right)$ can be represented as some 
linear combination of objects of the type (17) (when $x_{1}, \cdots, x_{n}$ do not coincide) it is enough to verify Wilson expansion only for $\varphi_{\{\nu\}}\left(x_{1} \cdots x_{n}\right)$. Let the positive numbers $\alpha_{1}, \cdots, \alpha_{n}$ be given $\left(\sum \alpha_{i}=1\right)$ and $x=\sum_{i=1}^{n} \alpha_{i} x_{i}$, $\xi_{i}=x_{i}-x$. The relative coordinates $\xi_{i}$ satisfy $\sum \alpha_{i} \xi_{i}=0$. Suppose also that $\xi_{i}{ }^{2} \neq 0$ and $\left(\xi_{i}-\xi_{j}\right)^{2} \neq 0$. Let $\xi_{i} \rightarrow 0$ remaining in the hyperplane $\sum \alpha_{i} \xi_{i}=0$. Evidently $: \varphi_{\left(\nu_{1}\right)}\left(x+\xi_{1}\right) \cdots \varphi_{\left(\nu_{n}\right)}\left(x+\xi_{n}\right):$ tends to $b_{\{\nu\}}(x)$. On the contrary the polylocal operator $\varphi_{\{\psi\}}\left(x+\xi_{1}, \cdots, x+\xi_{n}\right)$ does not tend to the corresponding composite field $B_{\{\nu\}}(x)$ since it is singular at $\xi_{1}=\cdots$ $=\xi_{n}$. The nature of this singularity is clear: when the vertices $x_{1}=x$ $+\xi_{1}, \cdots, x_{n}=x+\xi_{n}$ collide at $x$ in the expansion of $\varphi_{\{\nu\}}\left(x_{1}, \cdots, x_{n}\right)$ there arise new divergent subgraphs which are not suppressed by the initial $R$-operation. Still one can easily extract the regular part $\varphi_{\{\nu\}}^{\prime}\left(x+\xi_{1}\right.$, $\left.\cdots, x+\xi_{n}\right)$ from $\varphi_{\{\psi\}}\left(x+\xi_{1}, \cdots, x+\xi_{n}\right)$ just putting in analogy with (12) and (13)

$$
\begin{aligned}
\varphi_{\{\nu\}}^{\prime}\left(x+\xi_{1}, \cdots, x\right. & \left.+\xi_{n}\right)=E_{0}\left(S_{r}\right) \frac{1}{1+M E_{1}\left(S_{r}\right)} \\
& \times: \varphi_{\left(\nu_{1}\right)}\left(x+\xi_{1}\right) \cdots \varphi_{\left(\nu_{n}\right)}\left(x+\xi_{n}\right): .
\end{aligned}
$$

The polylocal operator $\varphi_{\{\nu\}}^{\prime}$ will be regular at $\xi_{1}=\cdots=\xi_{n}=0$ if $M$ "beforehand" makes the necessary substractions in the graphs of the right-hand side of (18) which become proper in the limit when the vertices $x+\xi_{1}$, $\cdots, x+\xi_{n}$ go to one point $x$. Moreover if in this limit the quantities like $M E_{1}\left(S_{r}\right): \varphi_{\left(\nu_{1}\right)}(x+\xi) \cdots \varphi_{\left.9 \nu_{n}\right)}\left(x+\xi_{n}\right)$ : tend to quantities like $M E_{1}\left(S_{r}\right)$ $\times b_{\{\nu\}}(x)$ one can assert that

$$
\lim _{\xi_{i} \rightarrow 0} \varphi_{\{\nu\}}^{\prime}\left(x+\xi_{1}, \cdots, x+\xi_{n}\right)=B_{\{\nu\}}(x) .
$$

Let us now construct such $M$. For any polylocal quantity $\varphi\left(x_{1}, \cdots, x_{n}\right)$ with $\operatorname{dim} \varphi \leqq a$ put

$$
M \varphi\left(x+\xi_{1}, \cdots, x+\xi_{n}\right)=\sum_{l+\Sigma\left|\lambda_{i}\right| \leqq a} K^{\{\lambda\}}\left(\xi_{1} \cdots \xi_{n}\right) b_{\{\lambda\}}(x)
$$

where

$$
K^{\{\lambda\}}\left(\xi_{1} \cdots \xi_{n}\right)=\frac{(-i)^{\Sigma\left|\lambda_{k}\right|}}{l !\left(\lambda_{1}\right) ! \cdots\left(\lambda_{2}\right) !}\left\langle\varphi\left(\xi_{1}, \cdots, \xi_{n}\right): \widetilde{\varphi}^{\left(\lambda_{1}\right)}(0) \cdots \widetilde{\varphi}^{\left(\lambda_{2}\right)}(0):\right\rangle^{\xi-\text { prop }} .
$$

Just comparing (20) and (21) with (8) and (9) one sees that $M$ possesses the necessary properties. Now we represent $\varphi_{\{\psi\}}^{\prime}\left(x+\xi_{1}, \cdots, x\right.$ 
$\left.+\xi_{n}\right)$ in the form

$$
\begin{aligned}
& \varphi_{\{\nu\}}^{\prime}\left(x+\xi_{1}, \cdots, x+\xi_{n}\right)=E_{0}\left(S_{r}\right): \varphi_{\left(\nu_{1}\right)}\left(x+\xi_{1}\right) \cdots \varphi_{\left(\nu_{n}\right)}\left(x+\xi_{n}\right):- \\
& \quad-E_{0}\left(S_{r}\right) \frac{1}{1+M E_{1}\left(S_{r}\right)} M E_{1}\left(S_{r}\right): \varphi_{\left(\nu_{1}\right)}\left(x+\xi_{1}\right) \cdots \varphi_{\left(\nu_{n}\right)}\left(x+\xi_{n}\right): .
\end{aligned}
$$

But $E_{0}\left(S_{r}\right): \varphi_{\left(\nu_{1}\right)} \cdots \varphi_{\left(\nu_{n}\right)}$ : is nothing else than the initial operator $\varphi_{\left\{{ }^{\prime}\right\}}\left(x+\xi_{1}\right.$, $\left.\cdots, x+\xi_{n}\right)$ and $M E_{1}\left(S_{r}\right): \varphi_{\left(\nu_{1}\right)}\left(x+\xi_{1}\right) \cdots \varphi_{\left(\nu_{n}\right)}\left(x+\xi_{n}\right)$ : can be expanded (according to (20)) into the sum of $b_{\{\lambda\}}(x)$, which under the action of $E_{0} / 1+M E_{1}$ will be transformed into the sum of $B_{\{\lambda\}}(x)$. Finally we have

$$
\begin{aligned}
\varphi_{\{\nu\}}(x & \left.+\xi_{1}, \cdots, x+\xi_{n}\right)=\sum_{i+\Sigma\left|\lambda_{i}\right| \leqq a} Q_{\{\nu\}}^{\{\lambda\}}\left(\xi_{1} \cdots \xi_{n}\right) B_{\left\{\alpha_{\}}\right.}^{a}(x) \\
& +B\left(x+\xi_{1}, \cdots, x+\xi_{n}\right)
\end{aligned}
$$

where

$$
B\left(x+\xi_{1}, \cdots, x+\xi_{n}\right)=\varphi_{\{\langle\}}^{\prime}\left(x+\xi_{1}, \cdots, x+\xi_{n}\right)-B_{\{\psi\}}^{a}(x)
$$

and

$$
\begin{aligned}
Q_{\{\nu\}}^{\{\lambda\}}\left(\xi_{1}, \cdots, \xi_{n}\right)=\delta_{\{\nu\}\{\lambda\}}+\frac{(-i)^{\Sigma\left|\lambda_{k}\right|}}{l !\left(\lambda_{1}\right) ! \cdots\left(\lambda_{l}\right) !} \times \\
\quad \times\left\langle E_{0}\left(S_{r}\right): \varphi_{\left(\nu_{1}\right)}\left(\xi_{1}\right) \cdots \varphi_{\left(\nu_{n}\right)}\left(\xi_{n}\right):: \tilde{\varphi}^{\left(\lambda_{1}\right)}(0) \cdots \widetilde{\varphi}^{\left(\lambda_{l}\right)}(0):\right\rangle^{\xi-p r o p} .
\end{aligned}
$$

Due to (19) $B\left(x+\xi_{1}, \cdots, x+\xi_{n}\right)$ is regular when $\xi_{i} \rightarrow 0$ and tends to zero in this limit. Relation (23) is just the Wilson expansion for $\varphi_{\{\psi\}}$.

\section{§5. Equations for Renormalized Green functions}

At last we give a hint of how the structure formula can be used to derive the equations for renormalized Green functions. Let the "bare" interaction Lagrangian be $\mathcal{L}(x)=-\frac{g}{24}: \varphi^{4}(x)$ :. First we redefine the subtraction operator $M$ in order to achieve a closer fit with the physical subtraction point on the mass shell. Let $F$ be a translation-invariant functional of the free field

$$
F=\sum_{l} \frac{1}{l !} \int f_{l}\left(x_{1} \cdots x_{l}\right): \varphi\left(x_{1}\right) \cdots \varphi\left(x_{l}\right): .
$$

Then

$$
M F=\frac{1}{2 !} \frac{\mu^{2} f_{2}\left(m^{2}\right)^{\text {prop }}-m^{2} f_{2}\left(\mu^{2}\right)^{\text {prop }}}{\mu^{2}-m^{2}} \int d x: \varphi^{2}(x):+
$$




$$
\begin{aligned}
& +\frac{1}{2 !} \frac{f_{2}\left(\mu^{2}\right)^{\text {prop }}-f_{2}\left(m^{2}\right)^{\text {prop }}}{\mu^{2}-m^{2}} \int d x: \partial^{\nu} \varphi(x) \partial_{\nu} \varphi(x): \\
& +\frac{1}{4 !} f_{4}\left(\operatorname{symm} \mu^{2}\right)^{\text {prop }} \int d x: \varphi^{4}(x):
\end{aligned}
$$

Here the symmetry point $\left(\operatorname{symm} \mu^{2}\right)$ is defined as in [3]. The extra parameter $\mu^{2}$ permits one to vary the field-strength renormalization constant; at $\mu^{2}=m^{2}$ one comes to the usual subtraction on the mass shell. For $x$-dependent functionals $\Psi(x)$ the subtraction $M$ is defined in such a way [10] that

$$
M \frac{\delta F}{\delta \varphi(x)}=\frac{\delta}{\delta \varphi(x)} M F
$$

Now let $S \rightarrow S+\delta S, M \rightarrow M+\delta M$ due to some variation of parameters of the theory. Then

\section{Lemma 3}

$$
\delta E\left(S_{r}\right)=E_{0}\left(S_{r}\right) \frac{1}{1+M E_{1}\left(S_{r}\right)}\left[\delta S-(\delta M) E_{2}\left(S_{r}\right)\right]
$$

So any derivative of the renormalized $S$-matrix is equal to some linear combination of composite fields which thus serves as Lowenstein generalized differentiation [3]. Relation (25) leads to the following equation of motion for composite fields $\varphi(x), \varphi^{3}(x)$ (Heisenberg counterpart of $\left.: \varphi^{3}(x):\right)$

$$
\left(\square+m^{2}\right) \varphi(x)=-\frac{g}{6} \varphi^{3}(x)
$$

In terms of Green functions $G$ this means (via Wilson expansion) that

$$
\begin{gathered}
\int d x\left\langle\left[\frac{g}{6} \varphi^{4}(x)+m^{2} \varphi^{2}(x)-\partial \varphi(x) \partial \varphi(x)\right] \varphi\left(x_{1}\right) \cdots \varphi\left(x_{N}\right)\right\rangle \\
=-i N G_{N}\left(x_{1} \cdots x_{N}\right) .
\end{gathered}
$$

The procedure now goes close to that of Lowenstein [3]. One applies the differential operator $D=a \frac{\partial}{\partial m^{2}}+b \frac{\partial}{\partial \mu^{2}}+c \frac{\partial}{\partial g}$ to $E_{0}\left(S_{r}\right), a, b$ and $c$ chosen in such a way that $D E_{0}\left(S_{r}\right)$ gives the same combination of com- 
posite fields as in the left-hand side of (26). Then using (26) one obtains the renormalization-group equations. On the other hand choosing $a, b$ and $c$ in such a way that $D E_{0}\left(S_{r}\right)$ reproduces the righthand side of Zimmermann identities and going to vacuum expectation values one gets the Callan-Symanzik equations.

Another way to use structure formula is to give the explicit expression of the renormalization constants (and their derivatives) in terms of renormalized Green functions. This leads to Fradkin equations [11] and to several similar but a bit more sophisticated relations [10].

\section{References}

[1] Wilson, K., Phys. Rev., 179 (1969), 1499.

Wilson, K. and Zimmermann, W., Comm. Math. Phys., 24 (1972), 87.

Zimmermann, W., Ann. of Phys., 77 (1973), 570.

Clark, T., Nucl. Phys., B 81 (1974), 263.

[2] Zimmermann, W., Ann. of Phys., 77 (1973), 536.

[3] see Lowenstein, J., Comm. Math. Phys., 24 (1971), 1 and references given there.

[4] Speer, E., Journ. Math. Phys., 9 (1968), 1404.

[5] 't Hooft, G. and Veltman, M., CERN-73-9, Geneva, 1973.

[6] Bogolubov, N. and Parasiuk, O., Acta Math., 97 (1956), 227.

[7] see Zavialov, O. I., Proc. of the Kyoto Conf. 1975. Lecture notes in physics, 39 (1975) and references given there.

[8] Anikin, S. A. and Zavialov, O. I., Dubna preprint D2-9259 (1975); Theor. and Math. Phys., 26 (1976), 162.

[9] Anikin, S. A., Zavialov, O. I., Theor. and Math. Phys. (in print).

[10] Zavialov, O. I., Theor. and Math. Phys. (in print).

[11] Fradkin, E., Proc. of Lebedev Physical Institute, USSR, 29 (1965). 